\title{
An Immersive Media Model of Amyotrophic Lateral Sclerosis
}

\author{
Federico Visi, Duncan Williams, Eduardo Miranda \\ Interdisciplinary Centre for Computer Music Research \\ (ICCMR), Plymouth University \\ Plymouth, UK \\ \{federico.visi, duncan.williams, eduardo.miranda\} \\ @plymouth.ac.uk
}

\author{
Giovanni Dothel \\ David Geffen School of Medicine \\ UCLA \\ Los Angeles, USA \\ giovanni.dothel@gmail.com
}

\section{INTRODUCTION}

Amyotrophic lateral sclerosis (ALS) also referred to as Motor Neurone Disease in the UK, is the most common of the five motor neuron diseases. Communicating about the behaviour of ALS with a wider audience remains one of the main goals of charities and associated organisations driving medical research and providing care for sufferers of ALS. This work presents a way of employing immersive media in order to illustrate the disease processes that characterise the progress of ALS and thus help raise awareness in the greater public.

\section{ABOUT ALS}

ALS is generally fatal within 5 years of onset. It affects approximately 1-2 people in every 100,000 (Cleveland et al. 2001). The disease is characterized by progressive degeneration of the upper and lower motor neurons of the spinal cord. This causes muscle weakness and atrophy throughout the body leading gradually to paralysis and death by respiratory failure. Several factors contribute to neuronal death, including Ribonucleic Acid (RNA) and protein dysfunctions. Due to its high complexity, the pathophysiology of the disease is yet to be completely clarified; however there is now a shared theory that the involvement of protein aggregation and deposit in neurons is linked to neuronal toxicity. Superoxide Dismutase-1 (SOD1) is a protein involved in cellular respiration. Genetic mutations or biochemical reactions (oxidation) lead to protein loss of function and misfolding in SOD1. This acquired aberrant conformation forms chainlike structures and aggregates in the neuronal body, the former most likely interacting with some of the physiological processes at the basis of cell survival (Bendotti et al. 2012, Chen et al. 2012, Tan et al. 2014). Today, gene therapy seems to be the most promising strategy for intervention with inherited ALS. Recent findings, depicting a deep heterogeneity of the disease, are paving the way for a multi-targeted therapeutic strategy (Robberecht \& Philips 2013).

\section{AN IMMERSIVE MEDIA MODEL}

The immersive media installation presented in this research utilizes published data obtained from Nuclear Magnetic Resonance studies of SOD1 protein (BMRB 2014). This data is mapped to sonic timbres and melodic patterns in a videosynchronous spatial speaker array that represents the nervous system. The accompanying video presents cues showing neural pathways as they are activated, whilst the associated neuromuscular junction is sounded on corresponding speakers in the array. Pitches are defined by mapping the average chemical shift of the $R$ group of each amino acid to notes on a tempered scale while the melody is defined by the amino-acidic sequence of the SOD1 protein. The envelope of the timbre of each note is defined by the nucleotides triples of each amino acid. The chemical shift of each nucleotide in the triple defines attack, decay and release of the note respectively. Over time, the timbre, rhythm, and spatialisation of these sonic patterns gradually changes to reflect the progress of the protein unfolding and aggregation. Melodic sequences change according to the mutation of the amino-acidic sequence. As the amino acids aggregate into fibrils, timbres become noisier to create additional spectral harmonics and, ultimately, introduce inharmonic distortion and dissonance. Video synchronicity gradually degrades as the nervous system loses control over the muscles and sclerosis hardens the affected nerves of the spinal cord. The spatialisation in the 3D speaker array, initially a fully immersive sound world, eventually shrinks completely, reflecting the patients' loss of movement and motor function. 


\section{REFERENCES}

Bendotti, C., Marino, M., Cheroni, C., Fontana, E., Crippa, V., Poletti, A., and De Biasi, S. (2012) Dysfunction of constitutive and inducible ubiquitin proteasome system in amyotrophic lateral sclerosis: implication for protein aggregation and immune response. Progress in Neurobiology, 97(2),101-26.

BMRB Biological Magnetic Resonance Data Bank. (2014) Statistics Calculated for All Chemical Shifts from Atoms in the 20 Common Amino Acids http://www.bmrb.wisc.edu/ref_info/statful.htm (retrieved on Feb 13th, 2014).
Chen, S., Zhang, X., Song, L., and Le, W. (2012) Autophagy dysregulation in amyotrophic lateral sclerosis. Brain Pathology, 22(1), 110-116.

Cleveland, D. W., and Rothstein, J. D. (2001) From Charcot to Lou Gehrig: deciphering selective motor neuron death in ALS. Nature Reviews. Neuroscience, 2(11), 806-19.

Robberecht, W., and Philips, T. (2013) The changing scene of amyotrophic lateral sclerosis. Nature Reviews Neuroscience 14(4), 248-264.

Tan, W., Pasinelli, P., and Trotti, D. (2014) Role of mitochondria in mutant SOD1 linked amyotrophic lateral sclerosis. Biochimica et Biophysica Acta, Feb 22. pii: S0925-4439(14)00048-9.

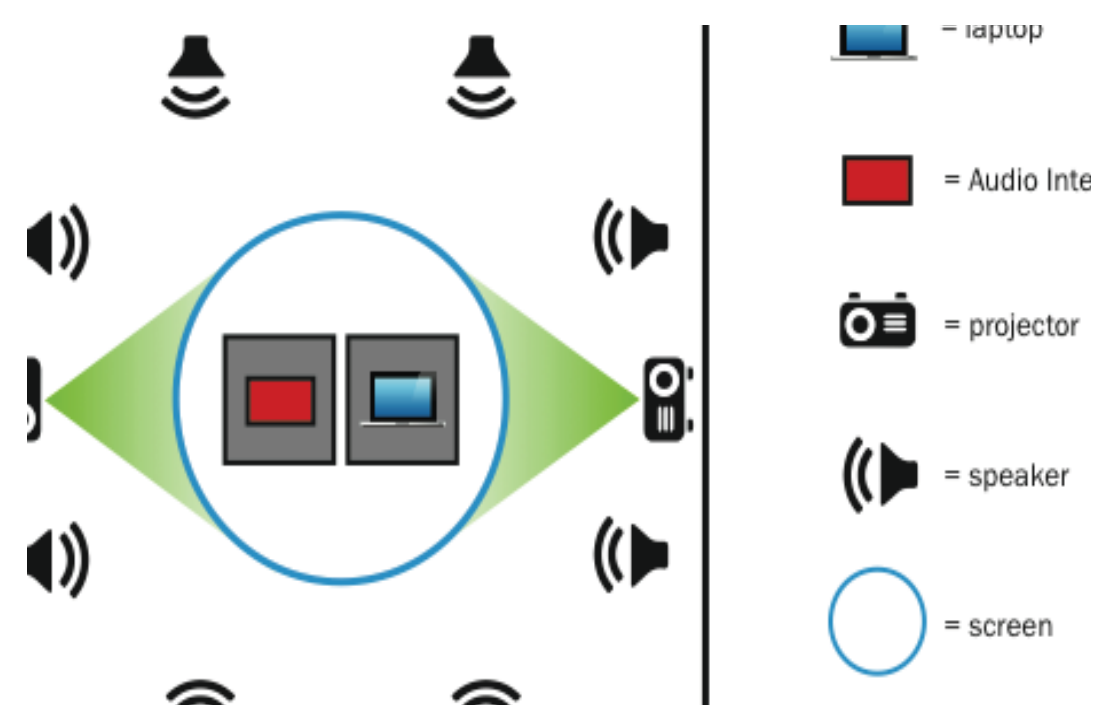

Figure 1: Floor plan

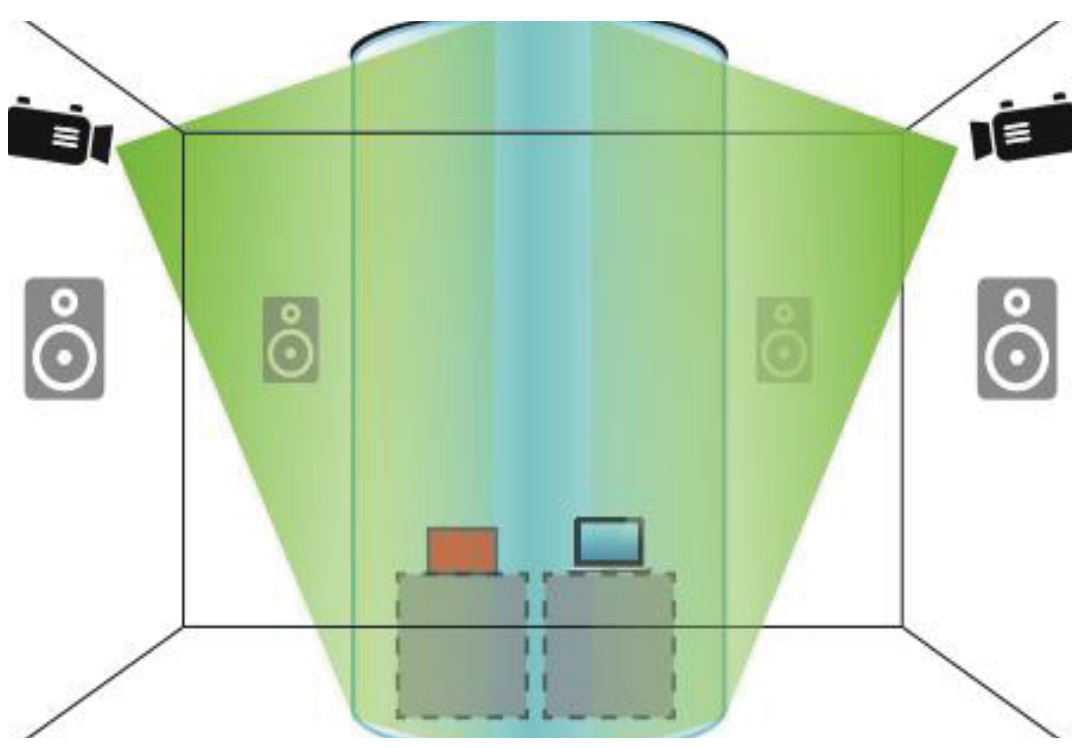

Figure 2: Side view 